Preprint of an article submitted for consideration in International Journal of Geometric Methods in Modern Physics (c) 2019 [copyright World Scientific Publishing Company]

[https://www.worldscientific.com/doi/abs/10.1142/S0219887819500713] 


\title{
Generating elastic solutions of the Einstein field equations from the Schwarzschild vacuum solution
}

\author{
Irene Brito \\ Centro de Matemática, Departamento de Matemática e Aplicações \\ Universidade do Minho, 4800-058 Guimarães, Portugal \\ e-mail: ireneb@math.uminho.pt
}

March 14, 2019

\begin{abstract}
The problem of generating solutions of the Einstein field equations with an elastic energy-momentum tensor from the Schwarzschild vacuum solution by means of conformal transformations is analysed. Applying the formulation of relativistic elasticity, suitable conformal factors are obtained for static and non-static elastic spacetime configurations and particular solutions are presented. This work shows that the technique used here permits generating new elastic matter solutions from a vacuum spacetime.
\end{abstract}

Keywords: relativistic elasticity; Einstein field equations; conformal transformations.

\section{Introduction}

The relativistic theory of elasticity is important and interesting for many astrophysical applications, e.g. for the study of deformations of massive stellar objects [1],[2], as in the description and modeling of neutron stars, whose solid crusts possess elastic properties [3]-[6]. The theory is also relevant for the study of anisotropy in pressures, a phenomenon occurring in many situations of equilibrium, which are of interest in astrophysics [7]. Different formulations of the relativistic theory of elasticity have been proposed and presented in the literature, see for instance [3],[4], [8], [9].

Due to the above reasons, obtaining exact solutions of the Einstein field equations (EFE) with elastic matter is a relevant and reasonable task. 
One approach in obtaining solutions of the EFE consists in generating new solutions from existing ones through conformal transformations. This technique was applied in [10]-[14] to construct perfect fluid solutions, where Hansraj [13] built a perfect fluid solution conformal to the Schwarzschild vacuum solution. Carot and Mas [15] and Tupper [16] obtained viscous fluid solutions by performing conformal transformations of vacuum solutions. In the context of relativistic elasticity, in [17] a new elastic solution was generated from a known shear-free spherically symmetric elastic solution.

Since it was shown that it is possible to obtain perfect fluid solutions and viscous fluid solutions from vacuum solutions and to construct new elastic fluids from existing ones, this motivates to investigate if it is possible to generate new elastic fluids from the Schwarzschild vacuum solution through conformal transformations. The aim of this paper is therefore to study this new problem of generating elastic solutions of the EFE from the Schwarzschild vacuum solution. For the given purpose, the Einstein field equations with an elastic energy-momentum tensor are derived for the conformal spacetime metric and the compatibility of the conformal factor with the elastic spacetime configuration is investigated. This problem is analysed separately for static and non-static conformal spacetime metrics. The reason for studying this problem separately, instead of considering the static case as a particular case of the non-static setting, is the following. In the static case it is possible to obtain a general solution for the conformal factor solving an ordinary differential equation, whereas in the non-static case, due to the complexity of the partial differential equations, only a particular class of solutions for the conformal factor is determined. Considering the subclass of static solutions of this particular class of non-static solutions would lead to physically not admissible solutions.

This paper is structured as follows. In Section 2, the problem of generating elastic solutions of the EFE from the Schwarzschild vacuum solution is set up and static conformal solutions are constructed in Subsection 2.1 and nonstatic conformal solutions, in Subsection 2.2. The matching problem with exterior spacetimes is also presented in those subsections. In Section 3 are summarized the main conclusions of this work. The Appendix contains a brief review of the relativistic theory of elasticity, based on the formulations presented in [4], [8] and [18], [19]. 


\section{Vacuum solution and conformal elastic solutions}

We will analyse if it is possible to generate an elastic fluid from the Schwarzschild vacuum solution in spherical symmetry by means of conformal transformations. Let $\bar{g}_{a b}$ denote the vacuum Schwarzschild metric given by the lineelement

$$
d s^{2}=-\left(1-\frac{2 M}{r}\right) d t^{2}+\left(1-\frac{2 M}{r}\right)^{-1} d r^{2}+r^{2} d \Omega^{2},
$$

where $d \Omega^{2}=d \theta^{2}+\sin ^{2} \theta d \phi^{2}$, and let $g_{a b}$ represent the metric of an elastic fluid in spherical symmetry. Then, the problem consists in determining a function $f^{2}$ such that

$$
g_{a b}=f^{2} \bar{g}_{a b}
$$

is a solution of the Einstein field equations for elastic matter. We will consider a static conformal metric with $f=f(r)$ in Subsection 2.1 and a non-static conformal metric with $f=f(t, r)$ in Subsection 2.2.

\subsection{Static conformal metric}

In this case, performing a conformal transformation of the Schwarzschild metric (1) with conformal factor $f^{2}(r)$, the aim is to investigate if

$$
g_{a b}=f^{2}(r) \bar{g}_{a b}
$$

with line-element

$$
d s^{2}=f^{2}(r)\left[-\left(1-\frac{2 M}{r}\right) d t^{2}+\left(1-\frac{2 M}{r}\right)^{-1} d r^{2}+r^{2} d \Omega^{2}\right]
$$

is a solution of the Einstein field equations for elastic matter.

In order to fit the spacetime $(\mathcal{M}, g)$ into the framework of relativistic elasticity and to describe the spacetime configuration, we will use the formulation presented in the Appendix.

Let $x^{a}, a=0,1,2,3$, be the spacetime coordinates: $\left(x^{0}, x^{1}, x^{2}, x^{3}\right)=(t, r, \theta, \phi)$, and let $y^{A}, A=1,2,3$, denote the material coordinates $\left(y^{1}, y^{2}, y^{3}\right)=(\tilde{r}, \tilde{\theta}, \tilde{\phi})$ in the material space $\mathcal{X}$. Here, we assume that the material metric $\gamma_{A B}$ in $\mathcal{X}$ is flat, its line-element being

$$
d \Sigma^{2}=d \tilde{r}^{2}+\tilde{r}^{2} d \tilde{\theta}^{2}+\tilde{r}^{2} \sin ^{2} \tilde{\theta} d \tilde{\phi}^{2} .
$$


Spherical symmetry implies that we can choose the following coordinates: $\tilde{r}=\tilde{r}(r), \quad \tilde{\theta}=\theta, \quad \tilde{\phi}=\phi$, leading to

$$
d \Sigma^{2}=d \tilde{r}^{2}+\tilde{r}^{2} d \theta^{2}+\tilde{r}^{2} \sin ^{2} \theta d \phi^{2} .
$$

Calculating the pulled-back material metric, one obtains

$$
\begin{aligned}
k_{b}^{a} & =g^{a c} k_{c b}=g^{a c} \gamma_{C B} y_{c}^{C} y_{b}^{B} \\
& =f^{-2}\left(1-\frac{2 M}{r}\right) \tilde{r}^{2} \delta_{1}^{a} \delta_{b}^{1}+f^{-2} r^{-2} \tilde{r}^{2}\left(\delta_{2}^{a} \delta_{b}^{2}+\delta_{3}^{a} \delta_{b}^{3}\right),
\end{aligned}
$$

$y_{c}^{C}$ being the relativistic deformation gradient defined in (104). The pulledback material metric has the eigenvalues

$$
\eta=f^{-2}\left(1-\frac{2 M}{r}\right) \tilde{r}^{2}, \quad \zeta=f^{-2} r^{-2} \tilde{r}^{2},
$$

where $\zeta$ has algebraic multiplicity two. Since the eigenvalues of the pulledback material metric are positive, one must consider $r>2 M$.

The invariants of $K_{b}^{a}=-u^{a} u_{b}+k_{b}^{a}$, where the velocity field of the matter is given by $u^{a}=\left(f^{-1}\left(1-\frac{2 M}{r}\right)^{-1 / 2}, 0,0,0\right)$, can be expressed in terms of the eigenvalues $\eta$ and $\zeta$ as

$$
\begin{aligned}
& I_{1}=\frac{1}{2}(\eta+2 \zeta-3), \\
& I_{2}=-\frac{1}{2}\left(\zeta^{2}+\eta+2 \zeta+2 \zeta \eta\right)+3, \\
& I_{3}=\frac{1}{2}\left(\eta \zeta^{2}-1\right)
\end{aligned}
$$

(cf. (109) and (110) with $\eta=n_{1}^{2}$ and $\zeta=n_{2}^{2}=n_{3}^{2}$ ). The components of the energy-momentum tensor for elastic matter (114) read

$$
\begin{aligned}
T_{0}^{0} & =-\rho, \\
T_{1}^{1} & =-\rho+\frac{\partial \rho}{\partial I_{3}} \eta \zeta^{2}-\frac{\partial \rho}{\partial I_{2}}(1+2 \zeta) \eta+\frac{\partial \rho}{\partial I_{1}} \eta \\
T_{2}^{2}=T_{3}^{3} & =-\rho+\frac{\partial \rho}{\partial I_{3}} \eta \zeta^{2}-\left[(1+\eta+2 \zeta) \frac{\partial \rho}{\partial I_{2}}-\frac{\partial \rho}{\partial I_{1}}\right] \zeta+\frac{\partial \rho}{\partial I_{2}} \zeta^{2},
\end{aligned}
$$

where the energy density is given by

$$
\rho=\epsilon v\left(I_{1}, I_{2}, I_{3}\right)=\epsilon_{0} \zeta \sqrt{\eta} v\left(I_{1}, I_{2}, I_{3}\right) .
$$


From (10)-(12) it follows that

$$
\begin{aligned}
& \frac{\partial \rho}{\partial \eta}=\frac{1}{2} \frac{\partial \rho}{\partial I_{1}}-\frac{1}{2}(1+2 \zeta) \frac{\partial \rho}{\partial I_{2}}+\frac{1}{2} \zeta^{2} \frac{\partial \rho}{\partial I_{3}}, \\
& \frac{\partial \rho}{\partial \zeta}=\frac{\partial \rho}{\partial I_{1}}-(1+\eta+\zeta) \frac{\partial \rho}{\partial I_{2}}+\eta \zeta \frac{\partial \rho}{\partial I_{3}} .
\end{aligned}
$$

These expressions allow to write the components of the energy-momentum tensor in the following way (using a process presented in [18], [19])

$$
\begin{aligned}
T_{0}^{0} & =-\epsilon v \\
T_{1}^{1} & =2 \eta \epsilon \frac{\partial v}{\partial \eta}, \\
T_{2}^{2}=T_{3}^{3} & =\zeta \epsilon \frac{\partial v}{\partial \zeta} .
\end{aligned}
$$

The Einstein field equations for the spherically symmetric elastic configuration with the spacetime metric (4) are then:

$G_{0}^{0}=T_{0}^{0}$ :

$$
\frac{2 f^{\prime \prime} f r(-2 M+r)+f^{\prime 2} r(2 M-r)+2 f^{\prime} f(-3 M+2 r)}{f^{4} r^{2}}=-\epsilon v
$$

$G_{1}^{1}=T_{1}^{1}$

$$
\frac{3 f^{\prime 2} r(-2 M+r)+2 f^{\prime} f(-3 M+2 r)}{f^{4} r^{2}}=2 \eta \epsilon \frac{\partial v}{\partial \eta}
$$

$G_{2}^{2}=T_{2}^{2}$ :

$$
\frac{f^{\prime 2}(2 M-r)+2 f^{\prime \prime} f(-2 M+r)+2 f^{\prime} f}{f^{4} r}=\zeta \epsilon \frac{\partial v}{\partial \zeta},
$$

where here and in the following text a prime stands for a derivative with respect to $r$.

The energy density, the radial pressure and tangential pressure are, respectively, expressed by

$$
\rho=\epsilon v, \quad p_{1}=2 \eta \epsilon \frac{\partial v}{\partial \eta}, \quad p_{2}=\zeta \epsilon \frac{\partial v}{\partial \zeta} .
$$


Dividing (23) and (24) through by (22) and setting $E \equiv \ln \eta$ and $Z \equiv \ln \zeta$ one obtains

$$
\begin{aligned}
\frac{\partial \ln v}{\partial E} & =-\frac{1}{2} \frac{3 f^{\prime 2} r(-2 M+r)+2 f f^{\prime}(-3 M+2 r)}{2 f^{\prime \prime} f r(-2 M+r)+f^{\prime 2} r(2 M-r)+2 f^{\prime} f(-3 M+2 r)} \\
\frac{\partial \ln v}{\partial Z} & =-\frac{r\left[f^{\prime 2}(2 M-r)+2 f^{\prime \prime} f(-2 M+r)+2 f^{\prime} f\right]}{2 f^{\prime \prime} f r(-2 M+r)+f^{\prime 2} r(2 M-r)+2 f^{\prime} f(-3 M+2 r)} .
\end{aligned}
$$

Let $F=\ln v$, then $F=F(E, Z)$, where $E$ and $Z$ are obtained from (9):

$$
\begin{aligned}
& E=-2 \ln f+\ln \left(1-\frac{2 M}{r}\right)+2 \ln \left(\tilde{r}^{\prime}\right) \\
& Z=-2 \ln f-2 \ln r+2 \ln \tilde{r} .
\end{aligned}
$$

Taking into account that $\frac{\partial F}{\partial E}$ and $\frac{\partial F}{\partial Z}$ are functions of $r$, one must guarantee that the condition $\frac{\partial^{2} F}{\partial E \partial Z}=\frac{\partial^{2} F}{\partial Z \partial E}$ is fulfilled in order for a constitutive equation to exist. This condition implies

$$
k\left(-\frac{f^{\prime}}{f}+\frac{M}{r(r-2 M)}+\frac{\tilde{r}^{\prime \prime}}{\tilde{r}^{\prime}}\right)=\left(-\frac{f^{\prime}}{f}+\frac{\tilde{r}^{\prime}}{\tilde{r}}-\frac{1}{r}\right),
$$

were $k \in \mathbb{R}^{+}$. Solving this ordinary differential equation gives

$$
f(r)=\bar{a} r^{\frac{2-k}{2(k-1)}}(2 M-r)^{\frac{k}{2(k-1)}} \tilde{r}^{\frac{1}{1-k}}\left(\tilde{r}^{\prime}\right)^{\frac{k}{k-1}},
$$

where $\bar{a} \in \mathbb{R}, k \in \mathbb{R}^{+} \backslash\{1\}$ and $f(r)$ must be a positive function, which is at least $\mathcal{C}^{2}$.

Therefore, in order to obtain static elastic solutions of the EFE which are conformal to the Schwarzschild vacuum solution, the conformal factor must be of the form

$$
f^{2}(r)=\bar{a}^{2} r^{\frac{2-k}{k-1}}(2 M-r)^{\frac{k}{k-1}} \tilde{r}^{\frac{2}{1-k}}\left(\tilde{r}^{\prime}\right)^{\frac{2 k}{k-1}},
$$

where $\bar{a} \in \mathbb{R}, k \in \mathbb{R}^{+} \backslash\{1\}$.

Next we will present two solutions, where the conformal factor has a particular form of (32), and analyse their physical plausibility. In the first example we will consider an elastic fluid having both radial and tangential pressure, whereas in the second example an elastic fluid with zero radial pressure and non-zero tangential pressure is considered. 
2.1.1 Example: $k=2, p_{1} \neq 0, p_{2} \neq 0$

Setting $k=2$ in (32), one obtains $f^{2}(r)=\bar{a}^{2} \frac{\left(\tilde{r}^{\prime}\right)^{4}}{\tilde{r}^{2}}(r-2 M)^{2}$. One can look for functions $\tilde{r}(r)$ such that $\frac{\left(\tilde{r}^{\prime}\right)^{4}}{\tilde{r}^{2}}=c$, where $c \in \mathbb{R}^{+}$. This condition leads to

$$
\tilde{r}(r)=\frac{\sqrt{c}}{4}\left(c_{1}-r\right)^{2},
$$

$c_{1}$ being an arbitrary constant. The conformal factor simplifies then and can be written as

$$
f^{2}(r)=a(r-2 M)^{2},
$$

with $a \in \mathbb{R}^{+}$. The elastic fluid metric, $g=f^{2}(r) \bar{g}$, is in this case given by the following line-element

$$
d s^{2}=a(r-2 M)^{2}\left[-\left(1-\frac{2 M}{r}\right) d t^{2}+\left(1-\frac{2 M}{r}\right)^{-1} d r^{2}+r^{2} d \Omega^{2}\right] .
$$

The energy density, radial pressure and tangential pressure are respectively

$$
\begin{aligned}
\rho & =\frac{3}{a r^{2}(r-2 M)^{2}}, \\
p_{1} & =\frac{7 r-6 M}{a r^{2}(r-2 M)^{3}}, \\
p_{2} & =\frac{1}{a r(r-2 M)^{3}} .
\end{aligned}
$$

The energy density satisfies $\rho \geq 0$ and the weak energy condition is satisfied for $r>3 M$ (the dominant energy condition is not fulfilled in this case, since $\rho-p_{1}<0$ for $\left.r>2 M\right)$.

\subsubsection{Example: $k=\frac{2}{5}, p_{1}=0, p_{2} \neq 0$}

Considering an elastic fluid with zero radial pressure, $p_{1}=0$, and non-zero tangential pressure, then equating (23) to zero leads to the following solution for $f(r)$ :

$$
f(r)=\frac{\bar{\alpha}}{r(r-2 M)^{\frac{1}{3}}}
$$

where $\bar{\alpha} \in \mathbb{R}^{+}$. This is a particular form of $(31)$, where $k=\frac{2}{5}$ and $\tilde{r}(r)$ must satisfy

$$
\frac{\tilde{r}^{\prime}(r)^{2}}{\tilde{r}(r)^{5}}=\frac{\bar{a}^{3}}{\bar{\alpha}^{3} r}
$$


which can be solved numerically (see Figure 1).

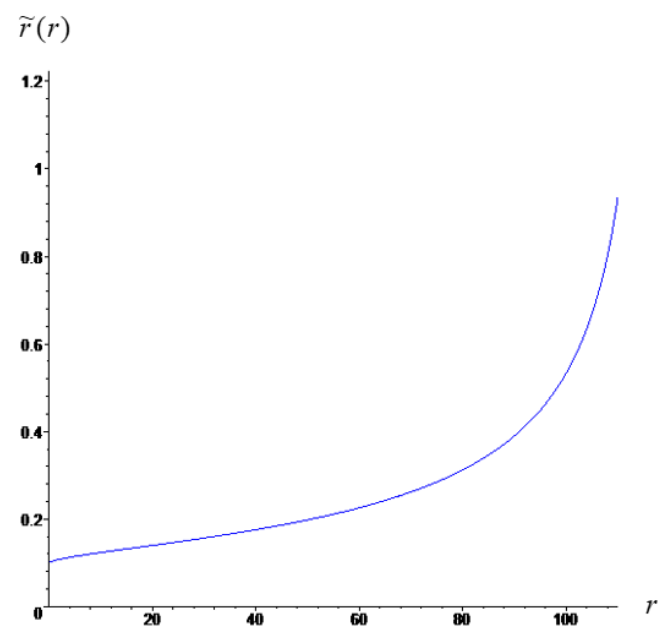

Figure 1: $\tilde{r}(r)$ for $\bar{a}=\bar{\alpha}=1$ with initial condition $\tilde{r}(0.1)=0.1$.

In this case, the spacetime metric $g$ is defined by the following line-element

$$
d s^{2}=\frac{\alpha}{r^{2}(r-2 M)^{\frac{2}{3}}}\left[-\left(1-\frac{2 M}{r}\right) d t^{2}+\left(1-\frac{2 M}{r}\right)^{-1} d r^{2}+r^{2} d \Omega^{2}\right]
$$

where $\alpha \in \mathbb{R}^{+}$.

The energy density and tangential pressure are, respectively, given by

$$
\begin{aligned}
\rho & =\frac{8(r-3 M)}{9 \alpha(r-2 M)^{1 / 3}}, \\
p_{2} & =\frac{4\left(4 r^{2}-21 M r+27 M^{2}\right)}{9 \alpha r(r-2 M)^{1 / 3}} .
\end{aligned}
$$

The energy density satisfies $\rho \geq 0$ for $r>3 M$ and the dominant energy condition $\left(\rho \geq 0, \rho \pm p_{2} \geq 0\right)$ is fulfilled for certain ranges of $r>3 M$.

\subsubsection{Junction of static spacetimes}

In [19] it is shown that shear free, static elastic solutions with line element

$$
d s^{2}=-a^{2}(r) d t^{2}+Y^{2}(r)\left(d r^{2}+d \Omega^{2}\right),
$$


can be matched to the Schwarzschild vacuum solution across a spherically symmetric static surface. Here we will show that the static conformal elastic solutions (4) can be matched to the solutions given by metric (44).

Consider the junction of the interior metric

$$
d s_{-}^{2}=f^{2}(r)\left[-\left(1-\frac{2 M}{r}\right) d t^{2}+\left(1-\frac{2 M}{r}\right)^{-1} d r^{2}+r^{2} d \Omega^{2}\right],
$$

with the exterior metric

$$
d s_{+}^{2}=-a^{2}(R) d t^{2}+Y^{2}(R)\left(d R^{2}+d \Omega^{2}\right),
$$

across a spherically symmetric surface $\Sigma$ of constant radius, where

$$
\Sigma_{-}=\left\{r=r_{\Sigma}\right\}, \Sigma_{+}=\left\{R=R_{\Sigma}\right\} .
$$

The signs - and + are used to denote, respectively, interior and exterior quantities. The normal vectors to the surface are

$$
n^{a-}=\frac{\sqrt{1-\frac{2 M}{r}}}{f} \partial_{r}, n^{a+}=\frac{1}{Y} \partial_{R} .
$$

The tangent space to $\Sigma$ can be written as

$$
T \Sigma^{ \pm}=\left\langle e_{1}^{ \pm}=\partial_{t}, e_{2}^{ \pm}=\partial_{\theta}, e_{3}^{ \pm}=\partial_{\phi}\right\rangle
$$

The first fundamental forms $q_{\alpha \beta}^{ \pm}=e_{\alpha}^{ \pm a} e_{\beta}^{ \pm b} g_{a b}^{ \pm}, \alpha, \beta=1,2,3$, at $\Sigma$ for both spacetimes are

$$
\begin{aligned}
& d \sigma_{-}^{2}=-f^{2}(r)\left(1-\frac{2 M}{r}\right) d t^{2}+f^{2}(r) r^{2} d \Omega^{2}, \\
& d \sigma_{+}^{2}=-a^{2}(R) d t^{2}+Y^{2}(R) d \Omega^{2}
\end{aligned}
$$

where all quantities must be evaluated on $\Sigma$. The first matching condition $q_{\alpha \beta}^{+}=q_{\alpha \beta}^{-}$leads to

$$
\begin{aligned}
& a^{2}(R) \stackrel{\Sigma}{=} f^{2}(r)\left(1-\frac{2 M}{r}\right), \\
& Y^{2}(R) \stackrel{\Sigma}{=} r^{2} f^{2}(r)
\end{aligned}
$$


where $\stackrel{\Sigma}{=}$ means that the equality holds only on the boundary $\Sigma$. The non-zero, independent components of the second fundamental forms $H_{\alpha \beta}^{ \pm}=$ $-n_{a}^{ \pm} e_{\alpha}^{ \pm b} \nabla_{b}^{ \pm} e_{\beta}^{ \pm a}$ at $\Sigma$ are

$$
\begin{aligned}
& H_{t t}^{-}=-\frac{a a^{\prime}}{Y}, \quad H_{\theta \theta}^{-}=Y^{\prime} \\
& H_{t t}^{+}=-\frac{f^{\prime} r^{2}-2 f^{\prime} r M+f M}{r^{2}} \sqrt{1-\frac{2 M}{r}}, \quad H_{\theta \theta}^{+}=r\left(f+f^{\prime} r\right) \sqrt{1-\frac{2 M}{r}},
\end{aligned}
$$

where all quantities must be evaluated on $\Sigma$. The equality of the second fundamental forms $H_{\alpha \beta}^{+}=H_{\alpha \beta}^{-}$imply

$$
\begin{aligned}
& \frac{a a^{\prime}}{Y} \stackrel{\Sigma}{=} \frac{f^{\prime} r^{2}-2 f^{\prime} r M+f M}{r^{2}} \sqrt{1-\frac{2 M}{r}}, \\
& Y^{\prime} \stackrel{\Sigma}{=} r\left(f+f^{\prime} r\right) \sqrt{1-\frac{2 M}{r}}
\end{aligned}
$$

Since

$$
p_{1}^{+}=2 \frac{a^{\prime}}{a} \frac{Y^{\prime}}{Y^{3}}+\frac{Y^{\prime 2}}{Y^{4}}-\frac{1}{Y^{2}},
$$

using (52), (53), (56), (57) to evaluate $p_{1}^{+}$on $\Sigma$, a straightforward calculation gives

$$
p_{1}^{+} \stackrel{\Sigma}{=} \frac{3 f^{\prime 2}\left(r^{2}-2 r M\right)+2 f f^{\prime}(2 r-3 M)}{r^{2} f^{4}},
$$

from where on concludes (c.f. (23)) that

$$
p_{1}^{+} \stackrel{\Sigma}{=} p_{1}^{-} .
$$

Calculating the mass $m^{-}$, one obtains

$$
m^{-}=\frac{f r}{2}-\frac{(r-2 M)\left(f^{\prime} r+f\right)^{2}}{2 f} .
$$

The mass of the exterior solution is given by

$$
m^{+}=\frac{Y}{2}\left(1-\frac{Y^{\prime 2}}{Y^{2}}\right) .
$$

Evaluating $m^{+}$at $\Sigma$ using (53) and (57) leads to

$$
m^{+} \stackrel{\Sigma}{=} m^{-} .
$$




\section{$2.2 \quad$ Non-static conformal metric}

In the non-static case, the aim is to determine $f^{2}(t, r)$ such that the metric $g_{a b}=f^{2}(t, r) \bar{g}_{a b}$ with line-element

$$
d s^{2}=f^{2}(t, r)\left[-\left(1-\frac{2 M}{r}\right) d t^{2}+\left(1-\frac{2 M}{r}\right)^{-1} d r^{2}+r^{2} d \Omega^{2}\right]
$$

is a solution of the EFE for elastic matter. In order to obtain a convenient expression for the conformal factor, here we follow a process analogous to one used in [17].

It is a known result, see for instance [14], [20], [21], that given two conformally related spacetime metrics $g=f^{2} \bar{g}$, the corresponding Einstein tensors can be related as follows

$$
\begin{aligned}
G_{a b}= & \bar{G}_{a b}-2 \bar{\nabla}_{b} \bar{\nabla}_{a} \ln f+2 \bar{\nabla}_{a} \ln f \bar{\nabla}_{b} \ln f \\
& +\bar{g}_{a b}\left(2 \bar{g}^{l m} \bar{\nabla}_{m} \bar{\nabla}_{l} \ln f+\bar{g}^{l m} \bar{\nabla}_{l} \ln f \bar{\nabla}_{m} \ln f\right),
\end{aligned}
$$

with $\bar{\nabla}_{a} \ln f=\nabla_{a} \ln f=\partial_{a} \ln f$. In the present case, since $\bar{G}_{a b}=0$ for the Schwarzschild vacuum metric $\bar{g}$ defined in (1), (65) can be written as

$$
G_{a b}=-2 \sigma_{a b}+\bar{g}_{a b}\left(2 \Delta_{2} \sigma+\Delta_{1} \sigma\right),
$$

where

$$
\begin{aligned}
& \sigma_{a b}=\bar{\nabla}_{b} \bar{\nabla}_{a} \ln f-\bar{\nabla}_{a} \ln f \bar{\nabla}_{b} \ln f, \\
& \Delta_{2} \sigma=\bar{g}^{l m} \bar{\nabla}_{m} \bar{\nabla}_{l} \ln f, \\
& \Delta_{1} \sigma=\bar{g}^{l m} \bar{\nabla}_{l} \ln f \bar{\nabla}_{m} \ln f .
\end{aligned}
$$

Then, the Einstein field equations $G_{a b}=T_{a b}$ and (66) imply

$$
T_{b}^{a}=G_{b}^{a}=\frac{1}{f^{2}}\left[-2 \bar{g}^{a m} \sigma_{m b}+\bar{g}_{b}^{a}\left(2 \Delta_{2} \sigma+\Delta_{1} \sigma\right)\right] .
$$

For the here considered metrics $\bar{g}$ and $g$ defined in (64), it follows from (68) that $\sigma_{10}=0$, which is equivalent to the condition

$$
M f \dot{f}+r(2 M-r)\left(\dot{f}^{\prime} f-2 f^{\prime} \dot{f}\right)=0,
$$

where $r \neq 2 M, f$ is a positive function and a dot represents a derivative with respect to $t$. Solving this equation, assuming that $f$ is in a separated form a product of functions of $t$ and $r$, gives

$$
f(t, r)=f_{1}(t) f_{2}(r), \text { where } \dot{f}_{1}=\frac{c_{1}}{f_{1}} \text { and } f_{2}^{\prime}=\frac{M f_{2}}{r(2 M-r)},
$$


$c_{1}$ being a constant. One concludes that a conformal factor of the form

$$
f^{2}(t, r)=\frac{a r(2 b t+c)}{2 M-r},
$$

where $a \in \mathbb{R}^{+}$and $b, c \in \mathbb{R}$, is a candidate to generate elastic fluid solutions from the Schwarzschild vacuum solution.

The corresponding conformal metric (64) reads

$$
d s^{2}=\frac{a r(2 b t+c)}{2 M-r}\left[-\left(1-\frac{2 M}{r}\right) d t^{2}+\left(1-\frac{2 M}{r}\right)^{-1} d r^{2}+r^{2} d \Omega^{2}\right] .
$$

It remains to analyse the compatibility of this class of solutions with an energy-momentum tensor corresponding to elastic matter and with the corresponding elastic spacetime configuration. For that purpose, we will establish the EFE in the relativistic elastic context, using again the approach presented in the Appendix.

Given the spacetime metric (72), the velocity field of the matter is defined by $u^{a}=\left(\frac{1}{\sqrt{-a(2 b t+c)}}, 0,0,0\right)$.

We assume that the line element of the material metric has the following form

$$
d \Sigma^{2}=q^{2}(r)\left[d r^{2}+r^{2}\left(d \theta^{2}+\sin ^{2} \theta d \phi^{2}\right)\right] .
$$

Then, the pulled-back material metric reads

$$
k_{b}^{a}=\frac{q^{2}}{f^{2}}\left[\left(1-\frac{2 M}{r}\right) \delta_{1}^{a} \delta_{b}^{1}+\delta_{2}^{a} \delta_{b}^{2}+\delta_{3}^{a} \delta_{b}^{3}\right],
$$

which has eigenvalues

$$
\eta=\frac{q^{2}}{f^{2}}\left(1-\frac{2 M}{r}\right) \text { and } \zeta=\frac{q^{2}}{f^{2}}
$$

where $\zeta$ has algebraic multiplicity two and $f^{2}=\frac{\operatorname{ar}(2 b t+c)}{2 M-r}$. Since the eigenvalues must be positive, it follows from (71) and from (75) that

$$
r>2 M \wedge 2 b t+c<0 \text {. }
$$

The EFE for elastic matter $G_{b}^{a}=T_{b}^{a}$, corresponding to the metric (72) 
with $T_{b}^{a}$ defined in (114), imply

$$
\begin{aligned}
\rho & =\epsilon v=\frac{3 M^{2}(2 b t+c)^{2}-3 b^{2} r^{4}}{a r^{4}(2 b t+c)^{3}} \\
p_{1} & =2 \eta \epsilon \frac{\partial v}{\partial \eta}=\frac{M(4 r-9 M)(2 b t+c)^{2}-3 b^{2} r^{4}}{a r^{4}(2 b t+c)^{3}} \\
p_{2} & =\zeta \epsilon \frac{\partial v}{\partial \zeta}=\frac{M(3 M-2 r)(2 b t+c)^{2}-3 b^{2} r^{4}}{a r^{4}(2 b t+c)^{3}}
\end{aligned}
$$

where $\rho$ is the energy density, $p_{1}$ the radial and $p_{2}$ the tangential pressure. Note that the last expressions depending on the eigenvalues are obtained from (114) in an analogous way as explained in Subsection 2.1 (or in [17], [18], [19]).

The weak energy condition is satisfied for certain ranges of $r$, however $\rho-$ $p_{2}<0$, so that the dominant energy condition is not satisfied.

As in the static case, dividing (78) and (79) by (77) and setting $E \equiv \ln \eta$ and $Z \equiv \ln \zeta$ gives

$$
\begin{aligned}
\frac{\partial \ln v}{\partial E} & =\frac{1}{2} \frac{\left(4 r M-9 M^{2}\right)(2 b t+c)^{2}-3 b^{2} r^{4}}{3 M^{2}(2 b t+c)^{2}-3 b^{2} r^{4}} \\
\frac{\partial \ln v}{\partial Z} & =\frac{\left(3 M^{2}-2 r M\right)(2 b t+c)^{2}-3 b^{2} r^{4}}{3 M^{2}(2 b t+c)^{2}-3 b^{2} r^{4}} .
\end{aligned}
$$

Let $F=\ln v$, then $F=F(E, Z)$, where $E$ and $Z$ are obtained from (75):

$$
\begin{aligned}
& E=2 \ln q+\ln \left(1-\frac{2 M}{r}\right)-\ln f^{2} \\
& Z=2 \ln q-\ln f^{2} .
\end{aligned}
$$

In order for a constitutive equation $v=v(\eta, \zeta)$, or equivalently $v=v(E, Z)$, to exist, we need to ensure that

$$
\frac{\partial^{2} \ln v}{\partial Z \partial E}=\frac{\partial^{2} \ln v}{\partial E \partial Z}
$$

We will see that this condition defines a function $q(r)$ for the material metric and imposes no further restriction on the conformal factor $f^{2}$. From 
expressions (82), (83) and applying the inverse function theorem, one gets

$$
\begin{aligned}
\frac{\partial t}{\partial E} & =\left(-\frac{q^{\prime}}{q}+\frac{M}{r^{2}-2 M r}\right) \frac{r(2 M-r)(2 b t+c)}{2 M b} \\
\frac{\partial t}{\partial Z} & =\left(\frac{q^{\prime}}{q}-\frac{2 M}{r(2 M-r)}\right) \frac{r(2 M-r)(2 b t+c)}{2 M b} \\
\frac{\partial r}{\partial E} & =-\frac{r(2 M-r)}{2 M} \\
\frac{\partial r}{\partial Z} & =\frac{r(2 M-r)}{2 M} .
\end{aligned}
$$

Since $\partial_{E}=\frac{\partial t}{\partial E} \partial_{t}+\frac{\partial r}{\partial E} \partial_{r}, \partial_{Z}=\frac{\partial t}{\partial Z} \partial_{t}+\frac{\partial r}{\partial Z} \partial_{r}$, one obtains from (84) together with (85)-(88)

$$
\frac{q^{\prime}}{q}=\frac{4(3 M-r)}{3 r(2 M-r)}
$$

yielding

$$
q(r)=\frac{c_{1} r^{2}}{\left[(r-2 M)^{2}\right]^{1 / 3}},
$$

where $c_{1}$ is an integration constant. This expression is not influenced by the constants $a, b, c$ of the conformal factor.

One concludes that it is possible to generate elastic non-static solutions of the Einstein field equations, which are conformal to the Schwarzschild vacuum solution. The solutions are given by (72), with $a>0 \wedge r>$ $2 M \wedge 2 b t+c<0$, and satisfy the weak energy condition for certain ranges of $r$. The corresponding material metric is given by (73) with $q(r)$ defined in (90).

Note that the conformal factor (71) is a particular solution of the partial differential equation (69). Setting $b=0$ in (72) would lead to a particular class of static solutions. These are, however, not physically admissible, since the energy density would be negative, as one concludes from (77) with $b=0$ and taking into account (76). The so obtained static solutions would coincide for $q(r)=1$ with a subclass of solutions of Section 2.1 for $\tilde{r}(r)=r$ and $k=\frac{1}{2}$. Next, a particular non-static solution is presented.

\subsubsection{Example: $a=1, b=-1 / 2, c=0$}

Setting $a=1, b=-1 / 2, c=0$ in (72), the metric $g$ conformal to the Schwarzschild vacuum metric, which is a solution of the EFE for elastic 
matter, is given by

$$
d s^{2}=\frac{t r}{r-2 M}\left[-\left(1-\frac{2 M}{r}\right) d t^{2}+\left(1-\frac{2 M}{r}\right)^{-1} d r^{2}+r^{2} d \Omega^{2}\right],
$$

and $r>2 M$. The line-element of $g$ can be simplified to

$$
d s^{2}=-t d t^{2}+t\left(1-\frac{2 M}{r}\right)^{-2} d r^{2}+t r^{2}\left(1-\frac{2 M}{r}\right)^{-1} d \Omega^{2} .
$$

The velocity field of the matter is given by $u^{a}=\left(t^{-1 / 2}, 0,0,0\right)$. The line element of the material metric is

$$
d \Sigma^{2}=q^{2}(r)\left[d r^{2}+r^{2}\left(d \theta^{2}+\sin ^{2} \theta d \phi^{2}\right)\right],
$$

with $q(r)$ defined in (90), and the pulled-back material metric reads

$$
k_{b}^{a}=\frac{q^{2}}{t}\left(1-\frac{2 M}{r}\right)\left[\left(1-\frac{2 M}{r}\right) \delta_{1}^{a} \delta_{b}^{1}+\delta_{2}^{a} \delta_{b}^{2}+\delta_{3}^{a} \delta_{b}^{3}\right] .
$$

The energy density, radial pressure and tangential pressure are, respectively,

$$
\begin{aligned}
\rho & =\frac{3 r^{4}-12 M^{2} t^{2}}{4 t^{3} r^{4}}, \\
p_{1} & =\frac{3 r^{4}+36 M^{2} t^{2}-16 M t^{2} r}{4 t^{3} r^{4}}, \\
p_{2} & =\frac{3 r^{4}-12 M^{2} t^{2}+8 M t^{2} r}{4 t^{3} r^{4}} .
\end{aligned}
$$

The weak energy condition is satisfied for certain ranges of $r$, however $\rho-$ $p_{2}<0$, so that the dominant energy condition is not satisfied.

\subsubsection{Junction of non-static spacetimes}

The non-static elastic solutions conformal to the Schwarzschild metric given by

$$
d s^{2}=f^{2}(t, r)\left[-\left(1-\frac{2 M}{r}\right) d t^{2}+\left(1-\frac{2 M}{r}\right)^{-1} d r^{2}+r^{2} d \Omega^{2}\right],
$$

with conformal factor defined in (71), can be matched to the exterior Vaidya solution given by

$$
d s^{2}=-\left(1-\frac{2 m(T)}{R}\right) d T^{2}-2 d T d R+R^{2} d \Omega^{2} .
$$


The matching conditions for the exterior Vaidya solution with interior general spherically symmetric spacetimes were derived e.g. in [22], [23]. In the present case, considering the parametrization $\{T=T(\lambda), R=R(\lambda)\}$ for the boundary $\Sigma^{+}$and $\{t=\lambda, r=r(\lambda)\}$ for the boundary $\Sigma^{-}$, one obtains the following matching conditions

$$
\begin{aligned}
R & \stackrel{\Sigma}{=} f r \\
\dot{T} & \stackrel{\Sigma}{=} \frac{f^{2}\left[\left(1-\frac{2 M}{r}\right)-\dot{r}\right]}{\dot{f} r+\left(1-\frac{2 M}{r}\right)\left(f^{\prime} r+f\right)}, \\
m(T) & \stackrel{\Sigma}{=} \frac{f r}{2}\left[1+\frac{(\dot{f} r)^{2}}{f^{2}\left(1-\frac{2 M}{r}\right)}-\frac{\left(f^{\prime} r+f\right)^{2}}{f^{2}}\left(1-\frac{2 M}{r}\right)\right], \\
\dot{r} & \stackrel{\Sigma}{=}-\left(1-\frac{2 M}{r}\right) \frac{p_{1}}{\rho},
\end{aligned}
$$

where $\rho$ and $p_{1}$ are defined, respectively, in (77) and (78) and a dot denotes differentiation with respect to $\lambda$. The expression on the right-hand side of (102) is equal to the mass of the interior solution. Notice that for a parametrization $\left\{t=\lambda, r=r_{\Sigma}\right\}$ for the boundary $\Sigma^{-}$one obtains $p_{1} \stackrel{\Sigma}{=} 0$ in (103).

\section{Conclusions}

In this paper we have investigated the problem of generating solutions of the EFE for elastic matter by applying conformal transformations to the Schwarzschild vacuum metric. This problem has been studied separately for static conformal spacetime metrics, where the conformal factor depends only on the radial coordinate, and for non-static conformal spacetime metrics, where the conformal factor depends also on the time coordinate. In the static configuration, two examples have been obtained: the first one represents an elastic fluid having both radial and tangential pressure and the second one corresponds to a fluid with vanishing radial pressure and non-zero tangential pressure. The dominant energy condition is fulfilled for the second example and the weak energy condition for the first example for certain ranges of the radial coordinate. In the non-static configuration, due to the complexity of the EFE, it is only possible to build up a class of particular solutions, which satisfy the weak energy condition for certain ranges of the radial coordinate. The static elastic conformal spacetimes can be joined to other elastic solutions, for which the matching with the Schwarzschild exterior 
solution is possible. The non-static elastic conformal spacetimes can be matched to the Vaidya exterior solution. One concludes that the approach used here allows generating elastic matter solutions from a vacuum solution.

\section{Acknowledgments}

The author thanks support from FCT ("Fundação para a Ciência e a Tecnologia"), through the projects UID/MAT/00013/2013 and PTDC/MATANA/1275/2014.

\section{Appendix}

Here we present a brief introduction to the relativistic theory of elasticity. The aim is to obtain the energy-momentum tensor modeling elastic matter, in order to determine the EFE in the relativistic elastic context. The reader is referred to the references [4], [8] and [18], [19] for more detailed aspects. Let $(\mathcal{M}, g)$ be a spacetime, where $\mathcal{M}$ is a four-dimensional manifold with Lorentz metric $g$ of signature $(-,+,+,+)$. Coordinates in $\mathcal{M}$ will be denoted by $x^{a}, a=0,1,2,3$. Suppose that the spacetime is filled with a continuous material. The material space $\mathcal{X}$ is a three-dimensional manifold, whose points represent the particles of the material. The material space is endowed with a Riemannian metric $\gamma$, the material metric, which measures the distance between particles in the locally relaxed state of the material. Material coordinates in $\mathcal{X}$ will be denoted by $y^{A}, A=1,2,3$. The spacetime configuration of the material is described by the configuration mapping $\psi: \mathcal{M} \longrightarrow \mathcal{X}$ through the fields $y^{A}=y^{A}\left(x^{a}\right), A=1,2,3$. The differential map $\psi_{*}: T_{p} \mathcal{M} \rightarrow T_{\psi(p)} \mathcal{X}$ gives rise to a rank 3 matrix with entries

$$
y_{b}^{A}=\frac{\partial y^{A}}{\partial x^{a}}, A=1,2,3, a=0,1,2,3,
$$

which is called the relativistic deformation gradient. The velocity field of the matter $u^{a}$, a future oriented, timelike unit vector field, which spans the one-dimensional Kernel of the relativistic deformation gradient, is defined by the conditions:

$$
u^{a} y_{a}^{A}=0, u^{a} u_{a}=-1, u^{0}>0 .
$$

The pulled-back material metric

$$
k_{a b}=\left(\psi^{*} \gamma\right)_{a b}=y_{a}^{A} y_{b}^{B} \gamma_{A B}
$$


is used to construct the strain tensor $s_{a b}=\frac{1}{2}\left(h_{a b}-k_{a b}\right)=\frac{1}{2}\left(g_{a b}-K_{a b}\right)$, where $h_{a b}=g_{a b}+u_{a} u_{b}$ and $K_{a b}=-u_{a} u_{b}+k_{a b}$. The material is said to be locally relaxed if the strain tensor vanishes. In the locally relaxed state, the operator $K^{a}{ }_{b}=k^{a}{ }_{b}-u^{a} u_{b}$ becomes the identity operator. Since $K^{a}{ }_{b} u^{b}=u^{a}$, one of its eigenvalue is 1 and the other three positive eigenvalues are the eigenvalues of $k_{b}^{a}$. One can use the orthonormal tetrad $\left\{u, e_{1}, e_{2}, e_{3}\right\}$, where $e_{1}, e_{2}, e_{3}$ are the spatial eigenvectors of $k_{b}^{a}$ and $u$ is the velocity field of the matter, which enables to write the metric $g_{a b}$ as

$$
g_{a b}=-u_{a} u_{b}+e_{1 a} e_{1 b}+e_{2 a} e_{2 b}+e_{3 a} e_{3 b}
$$

and the pulled-back material metric $k_{a b}$ as

$$
k_{a b}=n_{1}^{2} e_{1 a} e_{1 b}+n_{2}^{2} e_{2 a} e_{2 b}+n_{3}^{2} e_{3 a} e_{3 b},
$$

where $n_{1}^{2}, n_{2}^{2}, n_{3}^{2}$ are the eigenvalues of $k^{a}{ }_{b}$.

Assuming that the internal energy $v$ of an elastic deformation, accumulated in an infinitesimal portion of the material, is invariant with respect to its spacetime orientation, $v$ depends on three invariants of the strain tensor. Since the strain tensor is a linear function of $K$ and the identity operator, any three invariants $I_{1}, I_{2}, I_{3}$ of $K$ can be chosen, so that $v=v\left(I_{1}, I_{2}, I_{3}\right)$, which is the constitutive function of the material. Following [24] the invariants can be established as

$$
I_{1}=\frac{1}{2}(\operatorname{Tr} K-4), I_{2}=\frac{1}{4}\left[\operatorname{Tr} K^{2}-(\operatorname{Tr} K)^{2}\right]+3, I_{3}=\frac{1}{2}(\operatorname{det} K-1),
$$

where

$$
\begin{aligned}
& \operatorname{Tr} K=1+n_{1}^{2}+n_{2}^{2}+n_{3}^{2}, \operatorname{Tr} K^{2}=1+n_{1}^{4}+n_{2}^{4}+n_{3}^{4}, \\
& \operatorname{det} K=n_{1}^{2} n_{2}^{2} n_{3}^{2} .
\end{aligned}
$$

The energy density $\rho$ is defined by

$$
\rho=\epsilon v\left(I_{1}, I_{2}, I_{3}\right)=\epsilon_{0} \sqrt{\operatorname{det} K} v\left(I_{1}, I_{2}, I_{3}\right),
$$

where $\epsilon_{0}$ is the particle number density as measured in the material space, or rather, with respect to the volume form associated with $k_{a b}=\left(\psi^{*} \gamma\right)_{a b}$, and $\epsilon$ is that with respect to $h_{a b}$ (see e.g. [8],[24]). Since the invariants can be written in terms of the eigenvalues of $K$ using (110), the constitutive function can be viewed as a function of the eigenvalues, $v=v\left(n_{1}^{2}, n_{2}^{2}, n_{3}^{2}\right)$.

The energy-momentum tensor for elastic matter, when decomposed with respect to the matter velocity $u$, takes the form

$$
T_{a b}=\rho u_{a} u_{b}+p_{a b}=\rho u_{a} u_{b}+p h_{a b}+\pi_{a b},
$$


where $p_{a b}=p h_{a b}+\pi_{a b}$ is the pressure tensor, $p$ being the isotropic pressure and $\pi_{a b}$ the tracefree anisotropic pressure tensor. The pressure tensor $p_{a b}$ can be expressed as

$$
p_{a b}=p_{1} e_{1 a} e_{1 b}+p_{2} e_{2 a} e_{2 b}+p_{3} e_{3 a} e_{3 b}
$$

and the principal pressures $p_{1}, p_{2}, p_{3}$ satisfy $p_{1}+p_{2}+p_{3}=3 p$.

Following [24] (see also [18],[19]) the energy-momentum tensor for elastic matter can also be written as

$$
T_{b}^{a}=-\rho \delta_{b}^{a}+\frac{\partial \rho}{\partial I_{3}} \operatorname{det} K h_{b}^{a}-\left(\operatorname{Tr} K \frac{\partial \rho}{\partial I_{2}}-\frac{\partial \rho}{\partial I_{1}}\right) k_{b}^{a}+\frac{\partial \rho}{\partial I_{2}} k_{c}^{a} k_{b}^{c} .
$$

This tensor is obtained from the Lagrangian $\mathcal{L}=\sqrt{-g} \rho$, which is a function of $x^{a}, y^{A}$ and $y_{a}^{A}$. The corresponding Euler-Lagrange equations are given by $\frac{\partial \mathcal{L}}{\partial y^{A}}-\partial_{a}\left(\frac{\partial \mathcal{L}}{\partial y_{a}^{A}}\right)=0$, where $\frac{\partial \mathcal{L}}{\partial y_{a}^{A}}$ is sometimes called relativistic PiolaKirchhoff momentum tensor. Using Noether's theorem one constructs the canonical energy-momentum tensor

$$
\tilde{T}_{b}^{a}=\frac{1}{\sqrt{-g}} \frac{\partial \mathcal{L}}{\partial y_{a}^{A}} y_{b}^{A}-\delta_{b}^{a} \mathcal{L}
$$

which satisfies the energy-momentum conservation law $\nabla_{a} \tilde{T}^{a b}=0$, and the symmetric energy-momentum tensor is the negative of the canonical energymomentum tensor (see for instance [25]): $T_{a b}=-\tilde{T}_{a b}$. Note that (112) can be obtained from (115) (see [25]), where $p h_{a b}+\pi_{a b}=2 \frac{\partial \rho}{\partial G^{A B}} y_{a}^{A} y_{b}^{B}-\rho h_{a b}$, with $G^{A B}=y_{a}^{A} y_{b}^{B} g^{a b}$.

The interaction of the elastic material with the gravitational field is then described by the Einstein field equations $G^{a}{ }_{b}=T_{b}^{a}$, using units such that $8 \pi G=c=1$.

\section{References}

[1] Maugin, G.A., Ann. Inst. Henri Poincaré 15, 275 (1971).

[2] Carter, B., Quintana, H., The Astrophysical Journal 202, 511 (1975).

[3] Carter, B., Quintana, H., Proc. R. Soc. A, 331, 57 (1972).

[4] Karlovini, M., Samuelsson, L., Class. Quantum Grav. 20, 3613 (2003).

[5] Karlovini, M., Samuelsson, L., Zarroug, M., Class. Quantum Grav. 21, 1559 (2004). 
[6] Karlovini, M., Samuelsson, L., Class. Quantum Grav. 21, 4531 (2004).

[7] Herrera, L., et al., Phys. Rev D 69, 084026 (2004).

[8] Kijowski, J., Magli, G., J. Geom. Phys. 9, 207 (1992).

[9] Beig, R., Schmidt, B.G., Class. Quantum Grav. 20, 889 (2003).

[10] van den Bergh, N., J. Math. Phys. 27, 1076 (1986).

[11] van den Bergh, N., J. Math. Phys. 29, 1451 (1988).

[12] Loranger, J., Lake, K., Phys. Rev. D 78, 127501 (2008).

[13] Hansraj, S., Gen. Rel. Grav. 44, 125 (2012).

[14] Kumar, M.M., Il Nuovo Cimento LXIII, 559 (1969).

[15] Carot, J., Mas, L., J. Math. Phys. 27, 2336 (1986).

[16] Tupper, B.O.J., J. Math. Phys. 31, 1704 (1990).

[17] Brito, I., J. Math. Phys. 56, 092502 (2015).

[18] Brito, I., Carot, J., Vaz, E.G.L.R., Gen. Rel. Grav. 42, 2357 (2010).

[19] Brito, I., Carot, J., Vaz, E.G.L.R., Gen. Rel. Grav. 44, 287 (2012).

[20] Wald, R., General Relativity, University of Chicago Press, p.446 (1984).

[21] Eisenhart, L. Riemannian Geometry, Princeton University Press, p.89 (1968).

[22] Fayos, F., Jaen, X., Llanta, E., Senovilla, J.M.M, Phys. Rev. D 45, 2732 (1992).

[23] Carot, J., Tupper, B.O.J. Phys. Rev. D 59, 124017 (1999).

[24] Magli, G., Gen. Rel. Grav. 25, 441 (1993).

[25] Kijowski, J. and Magli, G., Preprint CPT-Luminy, 32/94, Marseille 207 (1994). 